\title{
Phase behavior of hard colloidal platelets using free energy calculations
}

\author{
Matthieu Marechal, ${ }^{1}$ Alejandro Cuetos, ${ }^{2}$ Bruno Martínez-Haya, ${ }^{2}$ and Marjolein Dijkstra ${ }^{1, a)}$ \\ ${ }^{1}$ Soft Condensed Matter, Debye Institute for NanoMaterials Science, Utrecht University, Princetonplein 5 , \\ 3561 RT Utrecht, The Netherlands \\ ${ }^{2}$ Departamento de Sistemas Físicos, Químicos y Naturales, Universidad Pablo de Olavide, 41013 Seville, \\ Spain
}

(Received 11 October 2010; accepted 17 January 2011; published online 1 March 2011)

\begin{abstract}
We investigate the phase behavior of a model for colloidal hard platelets and rigid discotic molecules: oblate hard spherocylinders (OHSC). We perform free energy calculations using Monte Carlo simulations to map out the phase diagram as a function of the aspect ratio $L / D$ of the particles. The phase diagram displays a stable isotropic phase, a nematic liquid crystal phase for $L / D \leq 0.12$, a columnar phase for $L / D \lesssim 0.3$, a tilted crystal phase for $L \lesssim 0.45$, and an aligned crystal phase for $L / D \gtrsim 0.45$. We compare the results to the known phase diagram of hard cut spheres. Thin cut spheres are almost cylinder-shaped, while the interactions between real discotic mesogens and colloidal platelets are more consistent with the toroidal rims of the OHSC. Since the shapes of the OHSC and the cut spheres are otherwise similar, the phase diagrams of the two types of particles are quite akin. However, the tilted crystal phase for OHSC, which is of a crystal type that is frequently found in experiments on disklike molecules, has not been found for hard cut spheres. Furthermore, although we have found a cubatic phase, it was shown to be definitely unstable, whereas the stability of the cubatic phase of cut spheres is still disputed. Finally, we also show that the phase boundaries differ significantly from those for cut spheres. These are remarkable consequences of a subtle change in particle shape, which show that for a detailed comparison with the phase behavior of experimental particles, the OHSC should be used as a model particle. (C) 2011 American Institute of Physics. [doi:10.1063/1.3552951]
\end{abstract}

\section{INTRODUCTION}

Disklike molecules are one of the most common particles that form liquid crystal phases, in particular columnar phases, which are interesting for technological applications. ${ }^{1}$ The most common model for hard disks or platelets that form a columnar phase is the cut sphere, ${ }^{2-7}$ which is a sphere where the top and bottom sections are chopped off. The resulting particle is shaped almost like a cylinder for thin cut spheres. Some concern has been expressed ${ }^{8}$ that the almost flat rims of such a model particle make it a less realistic model for the shape of disklike molecular mesogens. Furthermore, it is difficult to add attractions to the model that have the same shape anisotropy as the core. As there is no upper limit on the size of the molecules that can display liquid crystalline behavior, as long as sufficient thermal motion is present, also colloidal rods $^{9,10}$ and disks ${ }^{11,12}$ can form liquid crystalline phases. These colloids are stabilized either by charge repulsive or steric interactions, which tend to round off any sharp features of the underlying bare particles. Rodlike colloids are usually modeled as spherocylinders and disklike colloids as cut spheres. $^{2-4,6}$ While a spherocylinder has a smooth surface, the cut sphere has sharp edges and might not show the same phase behavior as the colloids it should correspond to. In short, the understanding of the phase behavior of both disklike colloids and disk-shaped molecular mesogens can likely be enhanced by considering an improved model for platelets.

a)Electronic mail: M.Dijkstra1@uu.nl.
In analog to the (prolate) spherocylinder, where the particle is defined as the volume within a certain distance from a line, an oblate spherocylinder can be defined by the volume within a certain distance to a disk. ${ }^{13}$ For prolate and oblate spherocylinders the particle-particle interactions are defined using the closest distance between the lines or disks, respectively. For hard particles an overlap occurs if this closest distance is smaller than a certain distance. This hard-core interaction can be replaced by a soft and/or attractive potential, that is a function of only one variable, the closest distance. Defining the potential in this way preserves the shape of the particles. ${ }^{14-16}$ In Refs. 8, 14, and 15, the phase diagram of oblate hard spherocylinders (OHSC) was tentatively explored using direct simulations. Three columnar phases were found for $0.1 \leq L / D \leq 0.5$, where $L$ is the thickness of the platelet and $D$ its diameter. At high densities the particles form columns that are interdigitated in the so-called interdigitated columnar phase $\left(D_{\mathrm{hi}}\right)$. As the density is decreased this $D_{\text {hi }}$ phase melts into a columnar phase $\left(D_{\text {ho }}\right)$ with local ordering within the columns, which melts into a disordered columnar phase $\left(D_{\mathrm{hd}}\right)$ at even lower density. In this paper, we study the phase behavior of oblate hard spherocylinders using free energy calculations. First, candidate crystal structures have to be found for which free energies can be calculated to determine their stability. In a recent paper, ${ }^{17}$ two of us, in collaboration with others, developed a new method to predict these candidate crystal structures, and we applied this method to a large variety of systems including the OHSC system. As a demonstration that these crystals were indeed 
stable, a part of the phase diagram of OHSC was shown as well, as determined from free energy calculations described in this paper. The two different crystals found using the method of Ref. 17 were indeed shown to be stable. In the present paper, we only briefly touch upon the method for finding the crystal structures for completeness, and focus in more detail on the simulation techniques used to map out the phase diagram. We will discuss the full phase behavior (in Ref. 17 only the crystals were discussed briefly) and compare it to the phase diagram of cut spheres. The phase diagram will also be compared to the earlier work of Refs. 8, 14, and 15 on hard oblate spherocylinders. We will show that the two newly found crystal phases effectively replace $D_{\text {hi }}$ in the phase diagram, in the sense that their combined regions of stability corresponds approximately to the region of stability of $D_{\text {hi }}$ in Refs. 8,14 , and 15 . To determine the continuous transition from $D_{\text {hd }}$ to $D_{\text {ho }}$ with similar accuracy as the other transitions, a proper finite size scaling analysis would be required (see Sec. IV), which is beyond the scope of the present study. Therefore, we have not distinguished between $D_{\text {hd }}$ and $D_{\text {ho }}$ and simply label the combined region of stability of the (hexagonal) columnar phase "col" in the phase diagram. In addition to the phase diagram, the maximum packing fractions of the various crystals are shown, which is of use to predict the phase behavior at infinite pressures. Well-packed structures can also serve as candidate crystal structures for other disklike systems (for instance repulsiveattractive disks $\left.{ }^{14-16}\right)$.

\section{MODEL}

We consider a system of hard platelets modeled as OHSC, which have been extensively described in previous works. ${ }^{8}, 13,14,18-23$ Therefore, we will only briefly describe the shape of our platelets. The OHSC consists of a flat cylindrical core with diameter $\sigma$ and height $L$, and a toroidal rim, with tube diameter $L$ (see Fig. 1). Consequently the total diameter of the OHSC is $D=L+\sigma$. We detect overlaps between OHSC using the efficient, although partially numerical algorithm, which was introduced in Ref. 8. Since that work, the
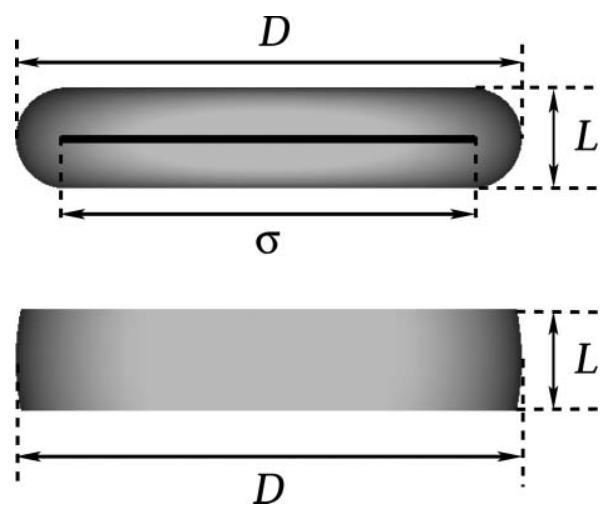

FIG. 1. Side view of the oblate hard spherocylinders (OHSC) considered in this study (top) compared to cut spheres (bottom) for $L / D=0.2$, where $L$ is the thickness of the platelets and $D$ the diameter. An OHSC is obtained by padding a disk of diameter $\sigma$, as indicated by the black line, with a layer of uniform thickness $L / 2$. overlap algorithm has been improved and some brief remarks on these changes are presented in the Appendix. As a result of these improvements, the algorithm is now faster and, more importantly, the presence or absence of an overlap can be exactly determined for all configurations that are likely to be encountered in our Monte Carlo (MC) simulations (see the Appendix for more details).

\section{METHODS}

In this section, we briefly reiterate the methods we employed to study the phase behavior using MC simulations, as these methods have been described extensively in Refs. 24 and 25 . First, we use the pressure annealing method ${ }^{17,25}$ to obtain candidate crystal structures. Here, we simulate a single unit cell of a crystal using a constant pressure MC in which the lengths of the edges of the simulation box and the angles between them can vary independently. ${ }^{26,27}$ Because we only simulate a single unit cell (2-6 particles in the simulation box), the fluctuations are large and many different crystal structures are probed. ${ }^{25} \mathrm{~A}$ fast compression finally results in well-packed crystal structures, among which is the stable crystal structure. Then we use the modified Einstein integration method, as described in Ref. 24 using simulations with many particles (1000-3000 particles typically), to obtain the free energy of these crystals at a certain reference density. As in the original Einstein integration technique, ${ }^{28}$ we couple each particle to its lattice position using a harmonic potential. Subsequently, the hard-core potential is replaced by an interpenetrable potential, which is slowly tuned from essentially hard to zero by decreasing the interaction strength $\gamma$. This interpenetrable interaction between each pair of particles, $i$ and $j$, is given by

$\beta \varphi\left(\mathbf{r}_{i j}, \mathbf{u}_{i}, \mathbf{u}_{j}, \gamma\right)= \begin{cases}\gamma\left(1-A\left(\rho_{i j} / L\right)^{2}\right), & \rho_{i j} \leq L, \\ 0, & \rho_{i j}>L,\end{cases}$

where $\mathbf{r}_{i j}=\mathbf{r}_{j}-\mathbf{r}_{i}, \rho_{i j}$ is equal to the closest distance between the disks in the middle of particles $i$ and $j, A$ is an adjustable parameter that is kept fixed at a value $A=0.5$ during the simulation, $\gamma$ is the integration parameter, and $\beta=1 / k_{B} T$, with $T$ being the temperature and $k_{B}$ being the Boltzmann's constant. We also use the Widom particle insertion method ${ }^{29}$ to obtain the chemical potential and therefore the free energy of the isotropic fluid and the nematic phases at a reference density. Third, we use the method by Bates and Frenkel ${ }^{30}$ adapted for particles with finite thickness, ${ }^{25}$ to get the free energy difference between the columnar phase at a reference density and the fluid phase at a lower density. Subsequently, we integrate the equation of state ${ }^{24}$ to obtain the free energy as a function of density. The equations of state for the crystal and the columnar phase were calculated using NPT MC simulations, where the length of the box vectors were allowed to change, but the angles between the box vectors were fixed at $90^{\circ}$. It was not necessary to allow these angles to adjust as the stable crystals all have rectangular unit cells. We checked that there was no difference between the equations of state that were obtained using NPT MC simulations with a rectangular box shape and simulations with a fully adjustable box shape. The equations of state for the isotropic 
fluid and the nematic phases were taken from Refs. 8 and 14 , which were augmented with results from additional NPT MC simulations at other densities and event-driven molecular dynamics (MD) simulations, which we will describe now. Two oblate spherocylinders overlap, when the closest distance between the two central disks (see Fig. 1) is shorter than $L$. Therefore, one can easily determine a collision event that occurs when the closest distance between two particles is equal to $L$. As a result, we can use event-driven MD simulations to investigate the phase behavior of hard oblate spherocylinders. The advantage of event-driven MD simulations over MC simulations is the fast accumulation of statistics of the pressure. ${ }^{31}$ In our NPT MC simulations the density changes by volume moves, which slows down equilibration of the density considerably, especially for large numbers of particles. In the MD simulations, the pressure is calculated from the momentum transfer during the collisions between the particles, ${ }^{31}$ and therefore comes for free with the integration of the equations of motion. Unfortunately, the shape of the simulation box needs to be known a priori, since the equilibration of the shape is just as slow or slower than the equilibration of the density. Therefore, the event-driven MD simulations were most useful for the isotropic fluid and nematic phases. For these phases, the equation of state from event-driven MD simulations and MC simulations gave the same results, although the MD results had a considerably lower statistical error. We implemented the event-driven MD simulation similarly as described in Ref. 32, except for the way we checked for grazing collisions (i.e., near misses). In an event-driven MD simulation of anisotropic particles, one searches for collisions on a grid in time; i.e., pairs of particles are moved forward in time and checked for overlap at regular intervals. In the event of a grazing collision, an overlap occurs in between two grid points, while no overlap is found at the grid points. Without a way to check for these grazing collisions, they will be missed, resulting in overlaps. In Ref. 32, a change in sign of the time derivative of the closest distance signals the presence of a minimum, which is a necessary condition for a grazing collision. Unfortunately, we were unable to find a robust and efficient way to calculate the time derivative of our numerical closest distance. Instead, we search for a minimum by comparing the closest distance at the middle of three grid points to the closest distance at the other two. If this closest distance is smaller than a certain cut off, we use a standard numerical routine to find the minimum and check whether this closest distance is smaller than $L$, in which case a collision is detected. Once a collision has been found we use a standard numerical root finder to locate the exact time of the collision.

To determine the isotropic-nematic (IN) coexistence, we use a comparatively simple method, devised for cut spheres by Fartaria and Sweatman: ${ }^{7}$ An NPT MC simulation is performed near the coexistence pressure $P_{\text {coex }}\left(P_{\text {coex }}\right.$ is first estimated by the pressure at which an isotropic fluid transforms into a nematic phase in a direct simulation). The histogram of observed values for the nematic order parameter $S_{2}$ is measured during this simulation. We stress that we did not employ the umbrella sampling technique to determine the histogram as was done in Ref. 7. This probability is double peaked near coexistence, where one peak at low $S_{2}$ corresponds to the isotropic phase and the other peak corresponds to the nematic phase. The applied pressure is equal to the pressure at coexistence when the heights of these two peaks are the same. This way of finding the coexistence pressure is rather slow, as a new simulation has to be performed for every pressure. However, we employ the histogram reweighing technique ${ }^{33}$ to determine the histogram at a pressure $P^{\prime}$, which is close to the pressure $P$ at which the simulation was performed, using

$$
\mathcal{P}\left(V, S_{2} \mid P^{\prime}\right) \propto \exp \left(-\beta\left(P^{\prime}-P\right) V\right) \mathcal{P}\left(V, S_{2} \mid P\right),
$$

where $\mathcal{P}\left(V, S_{2} \mid P\right)$ is the probability of finding a certain value $V$ for the volume and a certain value $S_{2}$ for the nematic order parameter. [In this equation, the proportionality factor is the ratio between the normalization constants of $\mathcal{P}\left(V, S_{2} \mid P^{\prime}\right)$ and $\mathcal{P}\left(V, S_{2} \mid P\right)$.] The Gibbs free energy is easily obtained using

$$
\frac{G\left(S_{2}\right)}{N k_{B} T}=-\ln \left[\mathcal{P}\left(V, S_{2} \mid P\right)\right]+\text { const. }
$$

For $L / D=0$, the $\mathcal{P}\left(S_{2}\right)$ always showed a significantly double peaked behavior with two maxima at $S_{2, I}$ and $S_{2, N}$ and the coexistence densities were simply defined as the densities at those values of $S_{2}$ :

$$
\rho_{I} \equiv \rho\left(S_{2, I}\right) \quad \text { and } \quad \rho_{N} \equiv \rho\left(S_{2, N}\right) .
$$

However, for small systems of OHSC with $L / D \geq 0.05$, the nematic order parameter of the isotropic phase is relatively high due to the presence of spinodallike nematic fluctuations in the isotropic fluid, caused by clusters with nematic order, ${ }^{7}$ whose sizes diverge as the spinodal is approached. ${ }^{34}$ As a result, the separation between the two peaks in the Gibbs free energy barrier is too small to distinguish them from statistical fluctuations. However, it is possible to obtain a flat distribution for a range of values of $S_{2}$ for a certain pressure; this pressure is defined as the coexistence pressure $P_{\text {coex }}$ for small systems. The definition (4) of the coexisting densities fails when $G\left(S_{2}\right)$ does not have a double peak. In order to perform finite size scaling, a definition for the coexisting densities that also works for these small system sizes is required. The simplest definition which gives the correct result for the infinite system is

$$
\rho_{\alpha} \equiv \int_{S_{2, \alpha}^{a}}^{S_{2, \alpha}^{b}} d S_{2} \rho\left(S_{2}\right) \mathcal{P}\left(S_{2}\right), \quad \text { for } \alpha=I, N,
$$

where $\mathcal{P}\left(S_{2}\right)=\int d V \mathcal{P}\left(V, S_{2} \mid P_{\text {coex }}\right)$ is the probability distribution of the nematic order parameter at coexistence, $\rho\left(S_{2}\right)$ $=\int d V(N / V) \mathcal{P}\left(V, S_{2} \mid P_{\text {coex }}\right)$ is the density as a function of the nematic order parameter, and $S_{2, \alpha}^{a}$ and $S_{2, \alpha}^{b}$ are the upper and lower boundaries of the nematic order parameter for phase $\alpha$. At coexistence, the probabilities of finding the system in the isotropic phase and the nematic phase should be equal, and $S_{2, N}^{a}=S_{2, I}^{b}$ was chosen accordingly, while the other boundaries are obviously, $S_{2, I}^{a}=0$ and $S_{2, N}^{b}=1$. This method of defining the coexistence densities was only required for $L / D \geq 0.05$. Both definitions of the coexistence densities give the same finite size corrected densities within the statistical error. The results for $L / D=0$ are of considerable interest, since $L / D=0$ is the limiting case for all disklike particles. Therefore, we spent more effort in 
determining the isotropic-nematic coexistence for $L / D=0$. Furthermore, the overlap criterion for infinitely thin platelets, i.e., $L / D=0$, is much faster. For these reasons, we have considered system sizes from $N=720$ to $N=4000$ for $L / D$ $=0$, while our largest system size for $L / D>0$ consisted of $N=2048$ particles. We also used a second method to obtain the isotropic-nematic coexistence: We simply measured the pressure from the momentum transfer during particle collisions in an event-driven MD and the chemical potential by the Widom particle insertion method. ${ }^{29}$ Subsequently, we fit lines through the chemical potential as a function of pressure and define the coexistence as the crossing point of these two lines. This would not have been possible using an NPT MC simulation, because (i) very long simulations would be required to sufficiently reduce the statistical error and (ii) the large system size that would be required to prevent the system from fluctuating between the isotropic and the nematic phases would slow down the NPT MC simulations even further.

Gibbs ensemble MC simulations are often used to study phase coexistence. ${ }^{35,36}$ In this method, two coexisting phases are simulated in two simulation boxes simultaneously. Moves that exchange particles and volume between the boxes at a fixed total number of particles $N$ and total volume $V$ ensure equal chemical potential and pressure between the two boxes. The densities and other properties of the coexisting phases can be determined by measuring the quantity of interest in each box separately. This method is quite often used for gasliquid phase coexistences, for which particle insertions into the higher density phase are relatively successful. However, when the temperature is raised such that the critical point is approached, the Gibbs ensemble method is abandoned for a histogram-based methods similar to the one we have used. ${ }^{36}$ Presumably, the Gibbs ensemble MC method is less accurate for weakly first order phase transitions. A possible argument, based on finite size scaling, might be as follows. First, we write the pressure as $P_{\alpha}\left(\rho_{\alpha}, N_{\alpha}\right)$ and the chemical potential as $\mu_{\alpha}\left(\rho_{\alpha}, N_{\alpha}\right)$ for the two phases, say $\alpha=I, I I$, where the finite size dependence has been made explicit by the second argument. For an equilibrated Gibbs ensemble simulation of systems $I$ and $I I$, the pressures and the chemical potentials in the separate boxes obey

$$
\begin{aligned}
& P_{I}\left(\rho_{I}, N_{I}\right)=P_{I I}\left(\rho_{I I}, N_{I I}\right), \\
& \mu_{I}\left(\rho_{I}, N_{I}\right)=\mu_{I I}\left(\rho_{I I}, N_{I I}\right),
\end{aligned}
$$

and, of course, $N_{I I}+N_{I}=N$ and $N_{I} / \rho_{I}+N_{I I} / \rho_{I I}=V_{I}$ $+V_{I I}=V$. The solution of this set of equations is strictly speaking only equal to the coexistence point in the thermodynamic limit $\left(N_{I}, N_{I I} \rightarrow \infty\right)$. However, if the resulting densities are close to the true coexisting densities and $N_{I}, N_{I I}$ are not too small, any thermodynamic variable $X_{\alpha}$ of phase $\alpha=I, I I$ can be approximated as

$$
X_{\alpha}\left(\rho_{\alpha}, N_{\alpha}\right) \simeq X_{\alpha}\left(\rho_{\alpha}^{c}\right)-\frac{X_{\alpha}^{(1)}}{N_{\alpha}}+\frac{\partial X_{\alpha}}{\partial \rho_{\alpha}}\left(\rho_{\alpha}-\rho_{\alpha}^{c}\right),
$$

where $X_{\alpha}\left(\rho_{\alpha}^{c}\right)=X_{\alpha}\left(\rho_{\alpha}^{c}, \infty\right), X_{\alpha}^{(1)}$ is a finite size scaling coefficient and $\rho_{\alpha}^{c}$ is the coexistence density of phase $\alpha$ for $N_{\alpha} \rightarrow \infty$. Inserting this approximation for the pressure and chemical potential at coexistence into Eq. (6) and solving for $\rho_{I}$ and $\rho_{I I}$, the densities obtained by the Gibbs ensemble method obey

$$
\frac{\rho_{I}-\rho_{I}^{c}}{\rho_{I}^{c}}=f_{I I}^{(1)} \rho_{I}^{c} \chi_{I}^{c} \frac{N_{I}^{-1}-N_{I I}^{-1}}{\rho_{I I}^{c}-\rho_{I}^{c}},
$$

and similarly for $\rho_{I I}$, where $f_{\alpha}^{(1)}=\rho_{\alpha} \mu_{\alpha}^{(1)}-P_{\alpha}^{(1)}$ for phase $\alpha$ and $\chi_{\alpha}^{c} \equiv \rho_{\alpha}^{-1}\left(\partial \rho_{\alpha} / \partial P\right)$, the isothermal compressibility of phase $\alpha$ at coexistence for $N_{\alpha} \rightarrow \infty$. In our case, $I$ denotes the isotropic phase and $I I$ the nematic phase. Using the measured $k_{B} T \chi_{I} D^{-3} \sim 0.02$ and estimated $\beta f_{I I}^{(1)} \rho_{I I} \sim 10$ [see Fig. 6(c)], $\chi_{I} f_{I I}^{(1)}$ can be estimated to be of order 1 for all aspect ratios. Equation (8) shows that Gibbs ensemble simulations for weakly first order transitions $\left(\rho_{I I}^{c}-\rho_{I}^{c} \ll \rho_{I}^{c}, \rho_{I I}^{c}\right)$ have to be performed on either very large systems or at a value of $N / V$ chosen such that $N_{I} \simeq N_{I I}$. However, the Gibbs ensemble method has been used previously ${ }^{5,37}$ to find the isotropic-nematic phase transition and, therefore, we also made an attempt to locate the isotropic-nematic coexistence using Gibbs ensemble simulations for OHSC with $L / D>0$. We compare the results to those of the other methods in Sec. IV.

\section{RESULTS}

\section{A. Packing}

We employ the pressure annealing method to find candidate crystal structures for the oblate hard spherocylinders. We find two crystal structures for $0<L / D<0.5$, a relative small number of candidate structures compared to the six structures found for bowls in Ref. 25. The particles in both crystal structures are stacked in columns and both crystals have a body-centered orthorhombic unit cell (see Fig. 2). One phase that we expected to find is the equivalent of the close packed phase for cut spheres, although slightly stretched to accommodate the slightly different shape of the OHSC. This phase consists of columns of platelets that are aligned along the $z$-axis; i.e., $\mathbf{u}_{i}$ is along $z$ for all particles $i$, where the $z$-direction is in the direction of the column. The columns are shifted with respect to each other in the $z$-direction, such that each column interdigitates with four of its neighboring columns, while each particle is at the same $z$-position as two particles in the other two neighboring columns. In the other crystal phase, particles again form columns. The particles in half of these columns tilt compared to the $z$-axis in the $x$ direction, where the $x$-axis is along the lattice vector perpendicular to $z$. The other half of the particles is tilted by the same angle in the $-x$-direction. In this way, each column interdigitates with all six of its neighbors. Some molecular mesogens
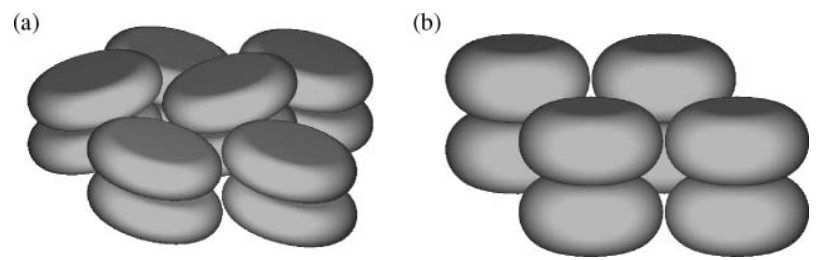

FIG. 2. The unit cells of the tilted crystal phase for $L=0.3 D$ (a) and the aligned crystal phase for $L=0.5 D(\mathrm{~b})$. 


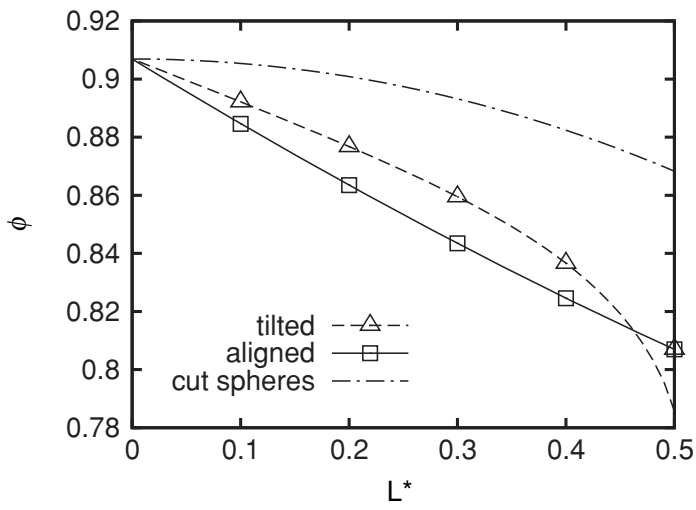

FIG. 3. Maximum packing fraction $\phi \equiv\left[(\pi / 6) L^{3}+\left(\pi^{2} / 8\right) \sigma L^{2}+\right.$ $\left.(\pi / 4) \sigma^{3}\right] N / V$ of the tilted and aligned crystal phases as a function of dimensionless thickness $L^{*} \equiv L / D$ for the oblate hard spherocylinders. The symbols denote the results of the pressure annealing. The analytical expression for the aligned crystal phases is denoted by the solid line, while, for the more complex tilted crystal phase, we used single high pressure runs with most of the degrees of freedom fixed, as described in the text (dashed line). The maximum packing fraction $\phi \equiv \frac{\pi}{4} L\left(D^{2}-L^{2} / 3\right) N / V$ of cut spheres as a function of $L^{*} \equiv L / D$ is shown for comparison (dot-dashed line).

also form tilted crystals, ${ }^{1}$ which were explained by calculating packing energies, ${ }^{38}$ but cut spheres do not. ${ }^{2}$

We determine the maximum packing fraction of these structures as described in Sec. III. The resulting packing fractions are shown in Fig. 3 as a function of $L$. The packing fraction is defined as $\phi=v N / V$, where

$$
v=\frac{\pi}{6} L^{3}+\frac{\pi^{2}}{8} \sigma L^{2}+\frac{\pi}{4} L \sigma^{2}
$$

is the volume of an OHSC. ${ }^{13}$ Additionally, we determined the lattice vectors (of the primitive unit cell) of the aligned crystal phase $\left(X_{\text {aligned }}\right)$ :

$$
\begin{gathered}
\mathbf{a}_{1}=D \hat{x}, \quad \mathbf{a}_{2}=L \hat{z}, \\
\mathbf{a}_{3}=\frac{D}{2} \hat{x}+\sqrt{\left(\sigma+\frac{L}{2} \sqrt{3}\right)^{2}-\left(\frac{D}{2}\right)^{2}} \hat{y}+\frac{L}{2} \hat{z},
\end{gathered}
$$

and the particles are aligned along $z$-direction. These lattice vectors result in a density:

$$
\rho D^{3}=D L \sqrt{\left(\sigma+\frac{L}{2} \sqrt{3}\right)^{2}-\left(\frac{D}{2}\right)^{2}} .
$$

We determined the lattice vectors and the direction vectors $\left(u_{ \pm}\right)$of the tilted crystal phase $\left(X_{\text {tilted }}\right)$ up to a free parameter $b$,

$$
\mathbf{a}_{1}=\left(\sigma u_{z}+L \sqrt{L^{2}-\sigma^{2} \sin ^{2} \theta_{0}}\right) \hat{x}, \quad \mathbf{a}_{2}=b \hat{y},
$$

$$
\mathbf{a}_{3}=\left(L / \cos \theta_{0}\right) \hat{z} \quad \text { and } \quad \mathbf{u}= \pm \sin \theta_{0} \hat{x}+\cos \theta_{0} \hat{z},
$$

where $\cos \left(2 \theta_{0}\right)=\sqrt{1-(L / \sigma)^{2}}$ and $\theta_{0}$ is the angle between the direction vectors and the $z$-axis. The free parameter $b$ cannot be determined analytically, since the closest distance between some of the neighboring particles in the tilted crystal can only be determined numerically. Instead, the lattice constant $b$ in the $y$-direction was determined in NPT MC simulations, which only include moves that change $b$. To be precise, we performed a single simulation of two particles at a pressure $\beta P D^{3}=10^{6}$ for each value of $L / D=0.01,0.02, \ldots, 0.5$. The resulting average maximum packing fractions for the $X_{\text {tilted }}$ phase are shown in Fig. 3 as a function of $L / D$ along with the analytical result for the $X_{\text {aligned }}$ phase as a solid and a dashed line, respectively. For comparison, we show the packing fraction of the close packed crystal for cut spheres in Fig. 3, which is clearly higher than the maximum packing fraction of OHSC for all aspect ratios.

\section{B. Isotropic-nematic coexistence}

While discotic liquid crystals usually form columnar phases, the most common and well-studied phase transition for colloidal disks is the IN coexistence. ${ }^{11,12,39-41}$ Surprisingly, the IN coexistence for platelets is only weakly dependent on size polydispersity. ${ }^{37}$ For these reasons, a relatively large amount of effort has been devoted to locating the isotropic-nematic coexistence for OHSC in the present paper by employing three different methods: (i) event-driven MD simulations, (ii) histogram method to determine the probability distribution of the nematic order parameter $S_{2}$, and (iii) direct NPT MC simulations. A fourth method that uses simulations in the Gibbs ensemble did not produce reliable results (see below). Using the first method, the coexistence between the isotropic fluid phase and the nematic phase were determined from the pressure and the chemical potential that were both directly measured in event-driven MD simulations. In Fig. 4, the chemical potential as a function of pressure is plotted for $L / D=0.1$ along with fits to the data. The pressure at coexistence defined by the intersection of these fits is $D^{3} P_{I N} / k_{B} T=32.0 \pm 0.1$. The packing fractions of the coexisting phases as obtained using local fits to the equation of state are shown by the pluses at $L / D=0.1$ in the

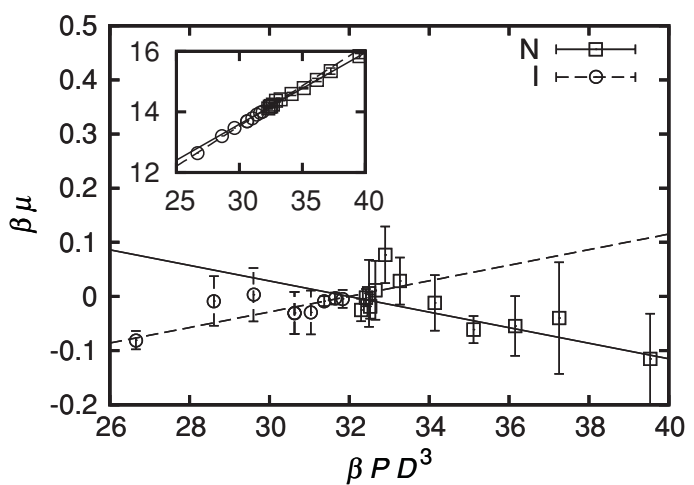

FIG. 4. The dimensionless chemical potential $(\beta \mu)$ vs the dimensionless pressure $\left(\beta P D^{3}\right)$ for the isotropic $(\mathrm{I})$ and the nematic phase $(\mathrm{N})$ near coexistence for a system of OHSC with $L / D=0.1$. A linear function of $P$ is subtracted from the measured chemical potential, which does not change the coexistence. The full chemical potential is shown in the inset, where it is defined such that the dimensionless ideal gas chemical potential reads $\log \left(\rho D^{3}\right)$. The error bars of the pressure data and the error bars of the chemical potential data for the isotropic phase are smaller than the symbol size. The lines are fits to the data. 


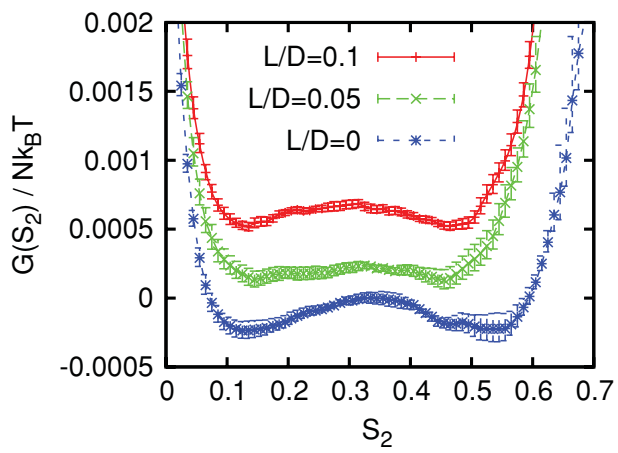

FIG. 5. The Gibbs free energy barriers as a function of $S_{2}$ [same as Fig. 6(a)] for different $L / D=00.05$ and $L=0.1$ at the same system size $N$ $=2048$. The barrier heights are $\Delta g^{*} \equiv \Delta G / N k_{B} T=0.002(1), 0.00010(5)$, and $0.00017(5)$ for $L / D=0,0.05$, and 0.1 , respectively.

phase diagram [Fig. 10(a)] and are tabulated in Table I. As explained in Sec. III, we have also used a second method of determining the isotropic-nematic coexistence, by finding the pressure for which the probability distribution function for the nematic order parameter $S_{2}$ has two equally high peaks. Subsequently, the Gibbs free energy $G\left(S_{2}\right) / N k_{B} T$ can be obtained from the probability distribution function at the coexistence pressure. Examples of $G\left(S_{2}\right)$ are shown in Fig. 5 for different aspect ratios $L / D$ and $N=2048$ particles, the largest system studied for OHSC with $L / D>0$. The barrier height $\Delta g^{*} \equiv \Delta G / N k_{B} T$ and the nematic order parameter $S_{2, N}$ of the nematic phase both decrease, when $L / D$ is increased from 0 to 0.05 , but little change is observed when $L / D$ is increased further to 0.1 . The barrier height $\Delta G$ is defined as the difference between the Gibbs free energy $G$ at the maximum and at the minima (see Fig. 5). The scaling of $G\left(S_{2}\right)$ with the size of the system is shown in Fig. 6(a) for $L / D=0$. Apparently, $G\left(S_{2}\right)$ depends only on system size for low $S_{2}$. This is a direct consequence of the well-known fact that the nematic order parameter of the isotropic phase has a larger finite size dependence $\left(S_{2} \sim 1 / \sqrt{N}\right)$ than $S_{2}$ of the nematic phase $\left(S_{2} \sim 1 / N\right){ }^{42}$ Interestingly, once the two minima in $G\left(S_{2}\right)$ become clearly separated, the dependence of the barrier between the minima on the system size seems to be very weak or even absent. This is caused by the absence of a clear isotropic-nematic interface in our simulations (such an interface would give rise to a $N^{-2 / 3}$ dependence). However, such an interface should form for systems larger than what we have studied (up to $N=10000$ ) and indeed it does form for colloidal platelets. ${ }^{41}$ We have performed finite size scaling for three values of $L / D=0,0.05$, and 0.1 . The results are shown in Figs. 6(b) and 6(c) again for $L / D=0$. Finally, the values for the nematic order parameter of the isotropic and nematic phases at coexistence can be fitted by well-known expressions (as discussed above). ${ }^{42}$

The methods described in Sec. III are quite elaborate and require considerable computing power. A much simpler, but less accurate approach is to perform NPT MC simulations, starting from the isotropic phase and increasing the pressure, as well as starting from the nematic phase and decreasing the pressure. The coexistence is approximately located at the (average) density and pressure at which the transition between
TABLE I. The isotropic-nematic phase transition: reduced densities of the isotropic phase $\rho_{I} D^{3}$, and the nematic phase $\rho_{N} D^{3}$, pressures $\beta P D^{3}$, and nematic order parameters $S_{2, N}$ at coexistence for OHSC and hard cut spheres with thickness $L$ and diameter $D$. The results for OHSC are obtained by direct simulation $(\dagger)$, by the calculation shown in Fig. 4 (*), and by the histogram method. The results for cut spheres are obtained from the literature; references are indicated in the second column.

\begin{tabular}{|c|c|c|c|c|c|}
\hline$L / D$ & $\rho_{I} D^{3}$ & $\rho_{N} D^{3}$ & $\beta P D^{3}$ & $S_{2, N}$ & \\
\hline \multicolumn{6}{|c|}{ OHSC } \\
\hline 0 & $4.029(6)$ & $4.340(10)$ & $15.304(7)$ & $0.521(6)$ & \\
\hline 0.05 & 3.957 (4) & $4.046(6)$ & $21.00(3)$ & $0.42(1)$ & \\
\hline 0.05 & \multicolumn{2}{|c|}{$3.97(13)^{\dagger}$} & $20.8(6)$ & & \\
\hline 0.07 & \multicolumn{2}{|c|}{$3.96(13)^{\dagger}$} & $24.2(2)$ & & \\
\hline 0.09 & \multicolumn{2}{|c|}{$3.91(14)^{\dagger}$} & $28.5(5)$ & & \\
\hline 0.1 & 3.968 (1) & $4.012(3)$ & $32.27(3)$ & $0.420(3)$ & \\
\hline $0.1^{*}$ & 3.94 & 4.01 & $32.0(1)$ & & \\
\hline 0.11 & \multicolumn{2}{|c|}{$4.06(8)^{\dagger}$} & $36.0(7)$ & & \\
\hline 0.12 & \multicolumn{2}{|c|}{$4.17(3)^{\dagger}$} & $42(1)$ & & \\
\hline$L / D$ & Ref. & $\rho_{I} D^{3}$ & $\rho_{N} D^{3}$ & $\beta P D^{3}$ & $S_{2, N}$ \\
\hline \multicolumn{6}{|c|}{ Cut spheres } \\
\hline 0 & 44 & 3.78 & 4.07 & & 0.37 \\
\hline 0 & 37 & $3.68(2)$ & $3.98(5)$ & & \\
\hline 0 & 45 & 3.344 & 3.680 & & 0.492 \\
\hline 0.001 & 7 & $4.02(4)$ & $4.30(4)$ & $15.4(1)$ & $0.54(8)$ \\
\hline 0.01 & 7 & $3.94(2)$ & $4.12(2)$ & $16.1(1)$ & $0.52(9)$ \\
\hline 0.04 & 7 & $3.85(2)$ & $4.04(2)$ & $19.6(1)$ & $0.47(4)$ \\
\hline 0.05 & 3 & 3.9 & 4.17 & $21.5(5)$ & \\
\hline $1 / 15$ & 4 & 3.86 & 4.11 & $24.8(1.0)$ & \\
\hline 0.07 & 7 & $3.88(1)$ & $4.05(1)$ & $26.0(3)$ & $0.63(4)$ \\
\hline 0.1 & 2 & 3.82 & 3.87 & & \\
\hline 0.1 & 5 & 4.11 & 4.26 & & \\
\hline 0.1 & 3 & 4.03 & 4.17 & 35.7 (3) & \\
\hline 0.1 & 7 & 4.17 (1) & $4.30(1)$ & $38.2(3)$ & $0.83(2)$ \\
\hline
\end{tabular}

the isotropic and nematic phases occurs in these simulations. ${ }^{2}$ We hardly find any hysteresis, and hence the transition in a compression and expansion run occurs at roughly the same density. Therefore, the width of the coexistence, i.e., the difference between the two coexisting densities, cannot be found using this method. In Fig. 7, we present the densities of the coexisting isotropic and nematic phases for varying aspect ratios of the OHSC. We find that the result for $L / D=0.1 \mathrm{ob}-$ tained from event-driven MD simulations matches well with the histogram method. Surprisingly, we also find good agreement between the results from the direct method and the histogram method within the statistical error bars, providing confidence in our results. The isotropic-nematic-columnar triple point lies somewhere between $L / D=0.12$ and 0.13 , since for $L / D=0.13$ a direct (spontaneous) transition from the isotropic phase to the columnar phase was observed. For comparison, we also plot the results for cut spheres from Ref. 7 based on the histogram method using umbrella sampling MC simulations. For small $L / D$, the effects of the sharpness of the circular edges of the cut spheres compared to the rounded edges of the OHSC should be minimal, especially for low packing fractions at which the isotropic-nematic coexistence occurs. Surprisingly, the coexisting densities are dissimilar for the two different model particles, as shown in Fig. 7, where we compare the phase boundaries for cut spheres with a certain $L / D$ as defined in Fig. 1 with those of OHSC with the 

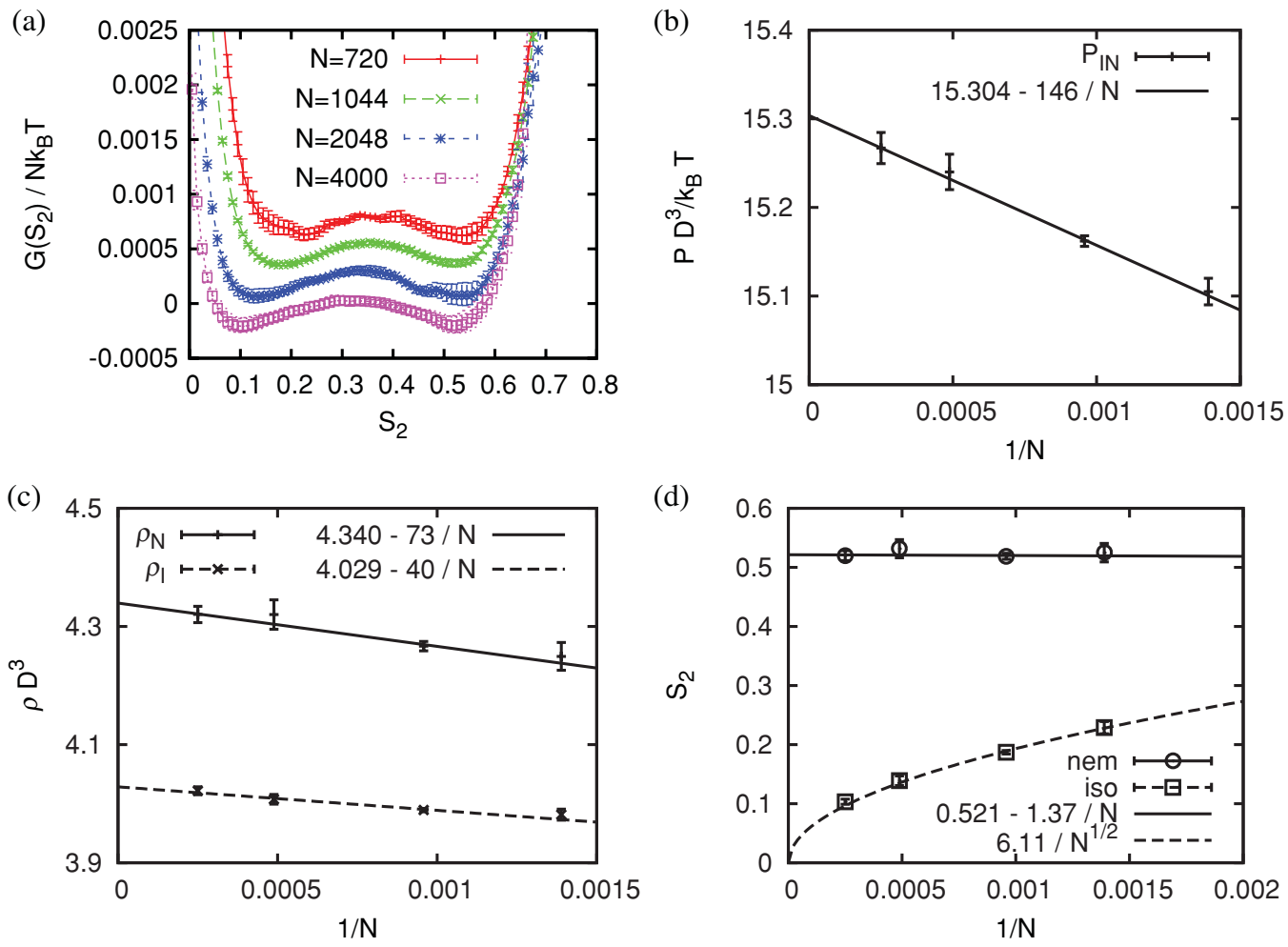

FIG. 6. Finite size effects of the isotropic-nematic coexistence for OHSC with $L / D=0$. (a) The dimensionless Gibbs free energy $G\left(S_{2}\right) / N k_{B} T$ as a function of the nematic order parameter $S_{2}$ for the four system sizes considered (from top to bottom) $N=720,1044,2048$, and 4000. [(b)-(d)] Finite size scaling of the coexistence pressure $\beta P D^{3}(\mathrm{~b})$, the coexisting densities of the isotropic phase $\rho_{I} D^{3}$ and the nematic phase $\rho_{N} D^{3}$ (c) and the nematic order parameter $S_{2}$ of the isotropic ("iso") and nematic ("nem") phases at coexistence (d).

same $L / D$. For a detailed comparison between different studies, we show literature results for cut spheres together with our results for OHSC in Table I. It is worth noting here that if we compare the phase boundaries for cut spheres with a certain $L / D$ with those for OHSC with the same thickness $L$ and the same particle volume $v$, we find essentially the same results. In fact, the volumes of the two types of particles are almost equal for the small values of $L / D$, at which the nematic phase is stable. Consequently, a comparison by packing fractions, instead of dimensionless densities $\rho D^{3}$ will also give the same result. In Ref. 7, systematic deviations inherent to the umbrella sampling method ${ }^{43}$ can play a role, while in our version of the histogram method no umbrella sampling was used. This could be a possible reason for the difference in the locations of the IN coexistence for OHSC and the results for cut spheres from Ref. 7. As the IN transition is weakly first order, the jump in density and nematic order parameter at the transition is not only very small, but also huge spinodallike nematic fluctuations ${ }^{34}$ appear in the isotropic fluid phase upon approaching the transition, which makes it extremely difficult to locate the transition. This difficulty is apparent from the large spread in literature values for the IN transition for cut spheres in Table. I. We would also like to mention that we performed extremely long runs in order to obtain histograms with enough statistics (more than $10^{8} \mathrm{MC}$ cycles typically, where a MC cycle consists of $N$ particle moves and 2 volume moves). Hence, equilibration problems seem unlikely. An alternative cause for the deviation might be that the weakly first order IN transition of platelets is extremely sensitive to the precise details of the particle shape, which requires further investigations. The minimum in the coexistence densities of the isotropic and nematic phases as a function $L / D$ that was observed for cut spheres ${ }^{7}$ is also found for OHSC in this paper. However, if coexisting packing fractions are plotted as a function of $L / D$ instead of coexisting densities, no minimum is found, because the $L / D$ dependence of the reduced volume of the OHSC dominates the dependence of the coexistence densities on $L / D$.

For completeness, we also attempted to find the coexistence using Gibbs ensemble simulations for OHSC with $L / D>0$. The resulting coexistence densities together with the coexistence as obtained using Gibbs ensemble simulations by Bates and Frenkel for $L / D=0$ (Ref. 37) are compared with the results from the histogram method in Fig. 8. When comparing Fig. 8 with Fig. 7, it can be seen that the differences between the Gibbs ensemble method and the histogram method are larger than the differences between the two particles shapes and the difference between the histogram method and the much simpler direct method. From Eq. (8) with $\left(\rho_{N}^{c}-\rho_{I}^{c}\right) / \rho_{I}^{c}$ less than 0.1 , the isotropic density as obtained from the Gibbs ensemble simulations has a relative deviation $\left(\rho_{I}-\rho_{I}^{c}\right) / \rho_{I}^{c}$ of a few percent or more for typical values of $N_{I}$ and $N_{N}$ used here and in Ref. 37, which explains the deviations from the histogram results in Fig. 8. These results clearly confirm that the Gibbs ensemble method should not be used to study weakly first order phase transitions.

Interestingly, the isotropic-nematic transition becomes more strongly first order for cut spheres with $L / D$ 


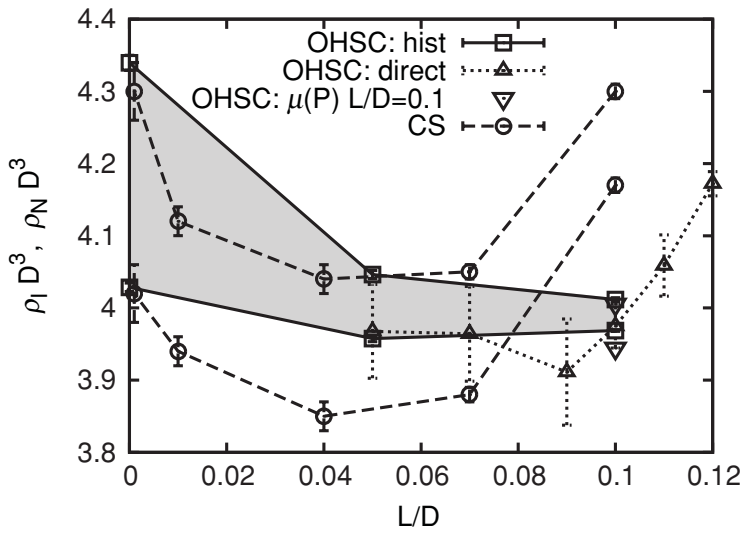

FIG. 7. A comparison between the isotropic-nematic (IN) phase coexistence for OHSC (squares and triangles) and the IN coexistence for cut spheres (CS) (circles) (Ref. 7). The results obtained from the histogram method (see Fig. 6) are denoted by the squares and the coexistence region is marked by the gray region. The triangles connected by the dotted line denote the results obtained using direct $N P T$ MC simulations with $N=1024$ and the inverted triangles denote the results obtained using event-driven MD simulations (see Fig. 4).

$=0.1,5,7$ such that the Gibbs ensemble MC method ${ }^{5}$ can be used more reliably: the coexistence densities from the Gibbs ensemble simulations ${ }^{5}$ are $\rho_{I} D^{3}=4.11$ and $\rho_{N} D^{3}$ $=4.26$, while using the biased histogram method $\rho_{I} D^{3}$ $=4.17(1)$ and $\rho_{N} D^{3}=4.30(1)$ were obtained. ${ }^{7}$ These values should be compared with the coexisting densities for the OHSC with $L / D=0.1$, which are $\rho_{I} D^{3}=3.968$ and $\rho_{I} D^{3}=4.012$. The agreement between the results for the cut spheres for $L / D=0.1$ signifies that the marked difference between our results for the OHSC for $L / D=0.1$ (see Table II) and the results for cut spheres for $L / D=0.1$ is not a result of equilibration or biasing problems in the various simulation methods, but rather a physical effect. The slightly higher value for the coexistence density of the nematic phase for cut spheres with $L / D=0.1$ compared to that for OHSC with $L / D=0.1$ has a rather large effect on the structure of the nematic phase at coexistence, see Fig. 9, which shows typ-

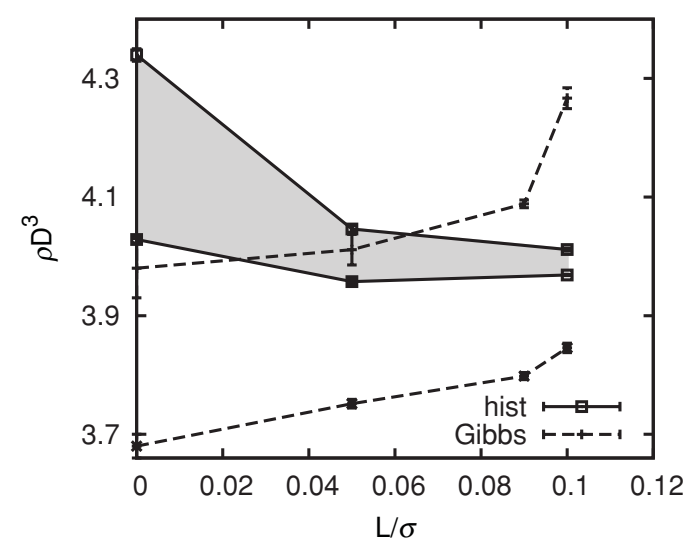

FIG. 8. A comparison between our results for the IN phase coexistence for OHSC obtained from the histogram method (solid line) and from Gibbs ensemble MC simulations (dashed line), completed with the results for the IN phase coexistence for $L / D=0$ from Ref. 37 based on the Gibbs ensemble method. The coexistence region between the solid lines is marked by the gray area.
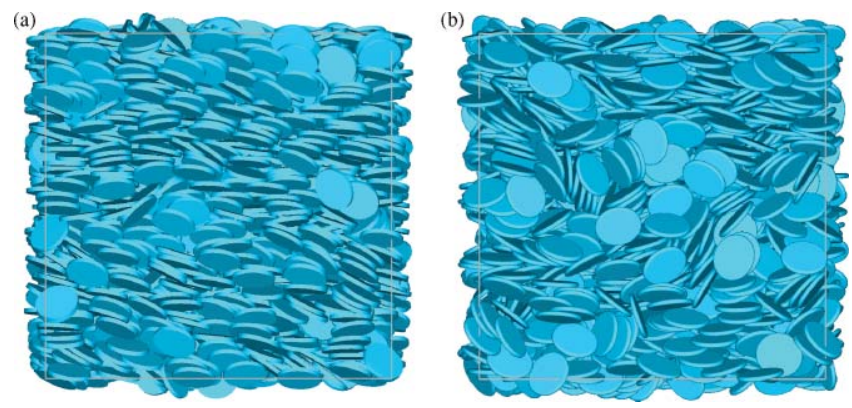

FIG. 9. Snapshots of the nematic phase near coexistence (a) for cut spheres with $L / D=0.1$ for $\phi=0.332$ or $\rho D^{3}=4.24$ and (b) for OHSC with $L / D=0.1$ for $\phi=0.300$ or $\rho D^{3}=3.99$. The nematic order parameter measured in the snapshot of (a) was $S_{2} \simeq 0.79$ and for (b) it was $S_{2} \simeq 0.46$.

ical configurations of the nematic phase of cut spheres and OHSC near coexistence with the isotropic phase. Remarkably, the nematic order parameter of the nematic phase is $S_{2} \simeq 0.79$ for cut spheres, while it is $S_{2} \simeq 0.46$ for OHSC, which is mainly due to the difference in density, not the difference in shape. Clearly, the subtle details of the particle shape have a huge effect on the IN phase coexistence.

\section{Phase diagram}

We now turn our attention to the remainder of the phase diagram. While the dimensionless densities $\rho D^{3}$ of the isotropic and nematic phases at coexistence between the two are nearly constant ( $\rho D^{3} \simeq 4$, see Fig. 7 and Table I), the transitions between the nematic phase and the columnar phase and between the columnar phase and the crystal occur at inconveniently large dimensionless densities for thin OHSC. Therefore, we will discuss the remainder of the phase diagram in terms of the packing fraction $\phi \equiv\left(\frac{\pi}{6} L^{3}+\frac{\pi^{2}}{8} \sigma L^{2}+\frac{\pi}{4} \sigma^{3}\right) \rho$ rather than the dimensionless density $\rho D^{3}$. Our simulations showed that the aligned crystal phase ( $\left.X_{\text {aligned }}\right)$ changed spontaneously into the $X_{\text {tilted }}$ crystal phase for $L / D \lesssim 0.4$ in a diffusionless transition (which is not a Martensitic transition, because there is no shear involved). Since this transition is diffusionless it is either second order or weakly first order, which allowed direct determination of the location of the transition. In order to determine the location of the transition, we performed two series of NPT MC simulations for $P^{*} \equiv \beta P D^{3}=75$ with increasing values of $L / D$ for the first series and decreasing values of $L / D$ for the second series of simulations. The first simulation of the first series started with an aligned crystal phase, while each next simulation was initiated from the final configuration of the previous simulation. When $L / D$ was decreased from $L / D=0.45$ to $L / D=0.44$, the system transformed into the tilted crystal phase, which was only possible because the aspect ratios of the simulation box were allowed to change. A similar series of simulations was performed for increasing $L / D$ starting from the tilted crystal phase. In this series of runs, the tilted crystal phase transformed into the aligned crystal phase between $L / D=0.46$ and $L / D=0.47$. Therefore, at some aspect ratio $L / D$ in between 0.44 and 0.47 the coexistence pressure must be $P^{*}=75$. At infinite pressure, the $P V$ term in the 

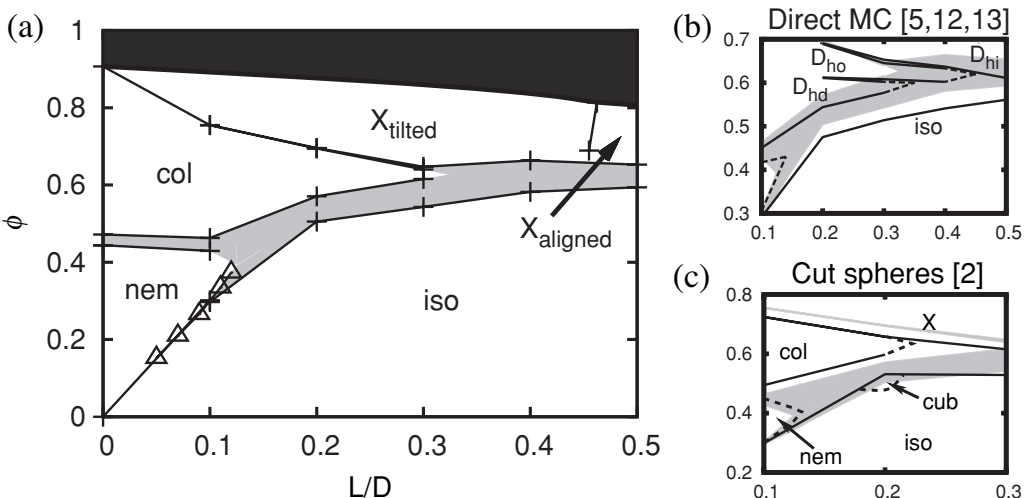

FIG. 10. Phase diagram in the packing fraction $(\phi)$ vs dimensionless thickness $(L / D)$ representation as obtained by free energy calculations (whenever possible) in this paper (a). The state points in the dark gray area are inaccessible since they lie above the maximum close packing line. $X_{\text {aligned }}$ and $X_{\text {tilted }}$, denote the aligned and tilted crystal structures as shown in Fig. 2, "iso" denotes the isotropic fluid, "nem" the nematic phase, and "col" the columnar phase. The solid lines are a guide to the eye, connecting coexistence points found in our simulations and, for $L / D=0$, from Ref. 30. The pluses at $L / D=0.1$ and the triangles are the results for the isotropic-nematic phase transition from different methods, which on this scale are indistinguishable from each other, see Fig. 7. In the panel on the right, we compare our results for OHSC (gray coexistence areas) to the phase diagrams in $(L / D)-\phi$ representation as obtained by direct simulations of OHSC (b) in Ref. 8 and free energy calculations on cut spheres (c) in Ref. 2. The various phases are labeled as in the previous works, and include, aside from the phases already mentioned, the three columnar phases $D_{\text {hd }}, D_{\text {ho }}$, and $D_{\text {hi }}$ (see text) from Ref. 8 and the cubatic (cub) and crystal (X) phases of the cut spheres from Ref. 2. Dashed lines are extrapolations (by us) of the coexistences of Refs. 8 and 2.

Gibbs free energy $G=F+P V$ is the only important term and, therefore, the transition between the $X_{\text {tilted }}$ and $X_{\text {aligned }}$ crystal phases must occur at the aspect ratio at which their close packed densities intersect (see Fig. 3), which was determined (numerically) to be $L / D \simeq 0.46232$. Aside from the nematic phase and the two crystal phases, that were already mentioned, the phase diagram also features a columnar phase. The coexistences between the isotropic fluid and the crystal and columnar phases were calculated using common tangent constructions on the free energy curves. The columnar-crystal coexistence pressure is the pressure at which the crystal melted and the corresponding coexistence densities are found using a fit to the equation of state. This means that these coexistence densities are actually lower bounds, except for $L / D=0.3$, where the columnar phase crystallized spontaneously with very little hysteresis. The free energy calculations at $L / D=0.3$ show that the columnar-tocrystal phase transition is only very weakly first order or even second order. The coexistences between the various phases for OHSC are shown in the phase diagram (Fig. 10) and tabulated in Table II.

We compare the phase diagram for OHSC obtained in this paper with the previously reported phase diagrams for OHSC of Ref. 8 and with the phase diagram for hard cut spheres of Ref. 2 in the panel on the right in Fig. 10. Figure 10(b) shows the phase diagram as obtained using direct simulations of OHSC in Ref. 8, along with the present coexistence regions (in gray). The present phase diagram shows similar trends as the phase diagram obtained from direct simulations: $:^{8,14,15}$ an isotropic fluid at the lowest densities for all aspect ratios $L / D$, a (discotic) nematic phase at low densities and aspect ratio $L / D \lesssim 0.1$, and phases in which the OHSC form aligned columns at high densities. However, using the pressure annealing method, we found two different crystals, $X_{\text {tilted }}$ and $X_{\text {aligned, }}$, which were found to be stable (using free energy calculations) in, respectively, the lower and higher $L / D$ regions of the phase diagram. Their combined region of stability is approximately, where the $D_{\text {hi }}$ phase of

TABLE II. Packing fractions $\phi$, dimensionless pressures $\beta P D^{3}$, and chemical potentials $\mu^{*}=\beta \mu-\ln \left(\mathcal{V} / D^{3}\right)$ of the coexisting phases for hard oblate spherocylinders with thickness $L$ and diameter $D$, where $\mathcal{V}$ is the thermal volume. See Table I for the isotropic-nematic coexistence. The coexisting densities marked by a ' $\dagger$ ' are the average of two coexisting densities determined by locating weakly first order or second order phase transitions in direct simulations. The transitions between the $X_{\text {tilted }}$ and $X_{\text {aligned }}$ crystal phases at $\beta P D^{3}=75$ and $\infty$ occur for OHSC with $L / D \simeq 0.46232$ and $L / D \simeq 0.455$, respectively (see text).

\begin{tabular}{|c|c|c|c|c|c|c|}
\hline$L / D$ & Phase 1 & Phase 2 & $\phi_{1}$ & $\phi_{2}$ & $\beta P D^{3}$ & $\mu^{*}$ \\
\hline$\overline{0.1}$ & nem & col & 0.4297 & 0.4646 & 66.96 & 21.2 \\
\hline 0.1 & col & $X_{\text {tilted }}$ & \multicolumn{2}{|c|}{$0.7551^{\dagger}$} & 325 & \\
\hline 0.2 & iso & $\mathrm{col}$ & 0.5051 & 0.5691 & 57.35 & 25.27 \\
\hline 0.2 & $\mathrm{col}$ & $X_{\text {tilted }}$ & \multicolumn{2}{|c|}{$0.695^{\dagger}$} & 115 & \\
\hline 0.3 & iso & col & 0.5433 & 0.6155 & 54.67 & 29.35 \\
\hline 0.3 & col & $X_{\text {tilted }}$ & \multicolumn{2}{|c|}{$0.6436^{\dagger}$} & 62 & \\
\hline $0.455(15)$ & $X_{\text {tilted }}$ & $X_{\text {aligned }}$ & 0.6891 & 0.6909 & 75 & \\
\hline 0.46232 & $X_{\text {tilted }}$ & $X_{\text {aligned }}$ & 0.8135 & 0.8135 & $\infty$ & $\infty$ \\
\hline 0.4 & iso & $X_{\text {tilted }}$ & 0.5818 & 0.6632 & 59.01 & 35.5 \\
\hline 0.5 & iso & $X_{\text {aligned }}$ & 0.5939 & 0.6527 & 53.59 & 37.36 \\
\hline
\end{tabular}


Ref. 8 is located. The simulations in Ref. 8 were initiated in a columnar phase, which is the reason that these crystal phases were not found. When re-examining the configurations obtained in the work of Ref. 8, we arrived at the conclusion that the configurations of the $D_{\mathrm{hi}}$ columnar phase from Ref. 8 are actually defective $X_{\text {tilted }}$ or $X_{\text {aligned }}$ crystals. We also observe from Fig. 10(b) that the strongly first order phase transitions, i.e., from the isotropic to the columnar or crystal phases, are shifted to higher densities in the present phase diagram compared to that of Ref. 8. This is to be expected, since Ref. 8 showed the location of the spontaneous melting of the columnar and crystalline phases. In Ref. 8, a distinction is made between two columnar phases, the $D_{\text {ho }}$ phase with long-range intercolumnar and intracolumnar correlations and the $D_{\text {hd }}$ phase, in which the columns become fluidlike. As already pointed out by De Gennes, ${ }^{46}$ the transition from $D_{\text {ho }}$ to $D_{\text {hd }}$, where the correlation length of the particles within a column becomes smaller than a certain threshold value (e.g., a few particle dimensions) is not sharp at all; the spatial correlations within a column will always have a finite correlation length. Hence, the transition will depend strongly on this precise threshold value, and in order to determine accurately the correlation length within the columns in simulations, a proper finite size scaling analysis is required, which is beyond the scope of the present study. Therefore, we simply labeled the combined region of stability of the columnar phases $D_{\mathrm{hd}}$ and $D_{\text {ho }}$ as "col" in Fig. 10(a). In summary, we find that our phase boundaries deviate from the earlier work in Ref. 8, but this can easily be explained by the fact that our phase diagram is obtained using free energy calculations and features crystal structures predicted by the pressure annealing method, while the previous phase diagram was obtained using direct simulations starting from a columnar phase.

The location of the isotropic-nematic coexistence for OHSC was already compared to that of cut spheres in Sec. IV B. The remainder of the phase diagram for hard cut spheres from Ref. 2 is compared with the present phase diagram for OHSC (in gray) in Fig. 10(c). We observe that the phase diagram of cut spheres is very similar to the one for OHSC, which is to be expected, since the shape of the cut sphere is not very different from the OHSC shape. There are a few interesting differences, such as the position of the isotropic-columnar-crystal triple point. This triple point is located in the range $0.1<L / D<0.2$ for cut spheres, where $L / D$ is the thickness-to-diameter ratio of cut spheres as defined in Fig. 1, while in our case it lies in the range 0.2 $<L / D<0.3$. This is caused by the increased stability of the crystal phase of cut spheres compared to the $X_{\text {tilted }}$ phase of OHSC, which can probably be related to the better packing of cut spheres in the corresponding crystal phase, see Fig. 3. The other difference between the phase diagram of OHSC and that of cut spheres is the absence of the cubatic phase for OHSC. Upon compression of an isotropic phase of OHSC with $L / D=0.2$, we did find a structure which resembled the cubatic phase, but it always transformed into the columnar phase (in an event-driven MD run). At packing fractions below that of the coexisting columnar phase, we have never found any evidence for the presence of a cubatic phase. The appearance of a cubatic phase in the phase diagram for cut spheres might be caused by the almost cylindrical shape of cut spheres with $L / D \simeq 0.2$ (see Fig. 1, which stabilizes stacks of around four particles, that are rotated by $90^{\circ}$ compared to adjacent stacks. However, the stability of the cubatic phase is still under debate. ${ }^{6}$

The phases present in our phase diagram represent some of the most commonly found phases in experiments on onecomponent thermotropic liquid crystals. ${ }^{47,48}$ Generalizations of the OHSC model (beyond the scope of this paper) to molecular shapes that are not cylindrically symmetric, to potentials that include (anisotropic) attractions, ${ }^{14-16}$ and finally to mixtures of different mesogens could lead to similar richness in phase behavior as experimentally observed for molecular liquid crystals. ${ }^{46}$ An example of the type of phase which could be found by a suitable generalization of our model is the nematic columnar phase. In this phase, disks stack to form columns that order like rods in a nematic phase. Such a phase has only been found experimentally for mixtures of disklike molecules with strong attractions between their central cores and various (typically) smaller molecules. ${ }^{49-53}$

\section{SUMMARY}

We determined the phase behavior of a model for hard cusp-free platelets: oblate hard spherocylinders (OHSC). Two crystal phases were found using the pressure annealing method. The first crystal structure for OHSC is similar to the one for cut spheres, albeit with a considerably lower close packed density. The second crystal structure that we predicted consists of columns of particles that are tilted with respect to the column and with respect to particles in other columns. Interestingly, tilted crystals are also commonly found for disklike molecules. ${ }^{1}$ However, only an aligned crystal phase was found for cut spheres, which indicates that the OHSC is a better model for molecular mesogens. The tilted crystal phase of OHSC also has a lower close packed density than the crystal phase of cut spheres. We used three different methods for tracing the phase boundary between the isotropic and the nematic phase. We find good correspondence between the three methods. However, we find larger differences than expected between the IN coexistence for OHSC and that of cut spheres ${ }^{7}$ even for thin platelets (except from $L / D \simeq 0$ ). However, the finding of Fartaria and Sweatman ${ }^{7}$ that the coexisting densities as a function of aspect ratio display a minimum also holds for OHSC. Subsequently, we calculated the full phase diagram of OHSC using free energy methods and compared it to the phase diagram of cut spheres and to earlier preliminary studies on OHSC using direct simulations for OHSC. $8,14,15$ The present paper confirms the trends found in the preliminary study, giving more accurate values for the location of the coexistence between the various phases. The improved accuracy will be necessary to apply the OHSC model in more complex studies, for instance, of platelets under gravity or studies of homogeneous or heterogeneous nucleation of platelets. Furthermore, the $D_{\text {hi }}$ phase that was found in Ref. 8 is most likely a defect-rich variant of one of our crystal phases. We also find that the overall shape of the phase diagram for OHSC is very similar to that of cut spheres, displaying stable isotropic, nematic, columnar, and crystal phases. 
Cut spheres have only one crystal phase (which is also stable for thick OHSC), while an additional stable crystal phase was found for OHSC. Furthermore, we did not find a stable cubatic phase which was found for cut spheres. These differences were explained on the basis of subtle differences in the shape of the respective particles. The isotropic-columnarcrystal triple point for the OHSC is shifted to lower $L$ compared to cut spheres, which can be understood from packing arguments. It is surprising that the phase behavior depends so sensitively on the precise details of the particle shape, which justifies the current paper and motivates the use of the OHSC as a model for particles that do not have sharp edges, like most experimental systems.

\section{ACKNOWLEDGMENTS}

M.D. and M.M. gratefully acknowledge financial support from a NWO-VICI grant and the High Potential programme (UU). A.C. and B.M.H. acknowledge support from research programmes of Andalucia (P09-FQM-4938) and Spain (CSD2009-00038). Matthew Dennison is acknowledged for useful discussions.

\section{APPENDIX: SOME NOTES ON THE OVERLAP ALGORITHM}

In Ref. 8, an efficient algorithm for finding the closest distance $\rho_{i j}$ between (infinitely thin) disks $i$ and $j$ was presented. Using this algorithm the overlap criterion for the OHSC simply corresponds to testing whether this distance $\rho_{i j}$ is larger or smaller than $L$. Furthermore, $\rho_{i j}$ can be used to define a soft interaction potential between two disklike particles. ${ }^{15}$ However, optimizations can be implemented in the case where simply the overlap between two OHSC is required. As a first optimization, the square of $\rho_{i j}$ can be calculated instead of $\rho_{i j}$ itself, which avoids the calculation a few (expensive) square roots. However, we will discuss distances rather than squared distances to simplify the notation. We will briefly describe the original algorithm (for further details see Ref. 8) and mark the points where further optimizations are possible. It should be noted that these optimizations are not required to successfully detect overlaps and, in fact, no difference between the two implementations could be detected apart from an increase in speed. In the first step of the overlap algorithm, it is checked whether the closest distance is between the edge of one of the disks $i$ and the face of the other disk $j$. Early on in this analytical step, the closest distance between disk $i$ and the plane in which disk $j$ lies is calculated. Clearly, if this distance is larger than $L$ no overlap can occur. In this case the other steps of the overlap algorithm can be avoided. If it is determined that the closest distance between the two disks is not between the edge of one disk and the face of the other, then the closest distance must be between the edges. It is here (and only here) that a numerical algorithm must be used. The iterative method of Ref. 8 is considerably faster than the standard minimization approach we have used to test against. In both methods, the distance between two points, $\mathbf{p}_{i}$ on the edge of disk $i$ and $\mathbf{p}_{j}$ on the edge of disk $j$, is minimized. The difference vector between the two points is $\Delta \mathbf{r} \equiv \mathbf{p}_{j}-\mathbf{p}_{j}$. A very simple optimization of any minimization method of this type is to stop whenever a distance $\Delta r \equiv|\Delta \mathbf{r}|$ is found which is smaller than $L$, because in this case the overlapping between the two OHSC is certain. Also the nonexistence of an overlap can be determined before the minimization procedure is complete. In order to do this, we have to consider the plane perpendicular to $\Delta \mathbf{r}$ at $\mathbf{p}_{i}+(L / 2) \Delta \mathbf{r} / \Delta r$. This plane is by construction tangent to particle $i$ at $\mathbf{p}_{i}$ and there is at least one point on the surface of particle $i$ on one side of it $\mathbf{p}_{i}+(L / 2) \Delta \mathbf{r} / \Delta r$ and a point on particle $j$ on the other side $\mathbf{p}_{j}-(L / 2) \Delta \mathbf{r} / \Delta r$ since $\Delta r>L$. So if the closest distances between disk $i$ and the plane and disk $j$ and the plane are larger than $L / 2$ there can be no overlap. The modifications to the numerical part of the overlap algorithm imply that the existence or nonexistence of overlap between two OHSC can be exactly determined for all configurations of particles whose surfaces are further apart than a certain $\epsilon$, which is the smallest possible precision which can be achieved using a given minimization method (all minimization methods have such an $\epsilon$; it is typically of the order of the square root of the machine precision ${ }^{54}$ ). For modern computers, this number is $\epsilon \simeq 10^{-11}$. Such small surface-tosurface distances are very unlikely to occur even once in a typical simulation, let alone that the incorrect assignment of (non)overlap to such a configuration would influence the results of the simulation noticeably.

As an aside, we note that if the algorithm iterates until the change of $\Delta r$ in a single iteration step is below a certain number $\epsilon_{\mathrm{it}}$ and $i$ and $j$ are nearly aligned and stacked on top of each other (a common configuration in the columnar or crystalline phases), then the error in the current estimates for $\mathbf{p}_{i}$ and $\mathbf{p}_{j}$ can be much larger than $\epsilon_{\mathrm{it}}$ : In that case, the iterative procedure gives the correct closest distance between the disks $\Delta r$ up to about $\epsilon_{\text {it }}$, but as $\Delta r$ depends only weakly on $\mathbf{p}_{i}$ and $\mathbf{p}_{j}$ for stacklike configurations, little effort is spent in determining these points in the iterative procedure. Therefore, a standard minimization technique was used for performing a collision in the event-driven MD, where $\mathbf{p}_{i}$ and $\mathbf{p}_{j}$ play an essential role. ${ }^{32}$

${ }^{1}$ C. D. Simpson, J. Wu, M. D. Watson, and K. Müllen, J. Mater. Chem. 14, 494 (2004).

${ }^{2}$ J. A. C. Veerman and D. Frenkel, Phys. Rev. A 45, 5632 (1992).

${ }^{3}$ S.-D. Zhang, P. A. Reynolds, and J. S. van Duijneveldt, J. Chem. Phys. 117, 9947 (2002).

${ }^{4}$ D. van der Beek, T. Schilling, and H. N. W. Lekkerkerker, J. Chem. Phys. 121, 5423 (2004).

${ }^{5}$ M. M. Piñeiro, A. Galindo, and A. O. Parry, Soft Matter 3, 768 (2007).

${ }^{6}$ P. D. Duncan, M. Dennison, A. J. Masters, and M. R. Wilson, Phys. Rev. E 79, 031702 (2009).

${ }^{7}$ R. P. S. Fartaria and M. B. Sweatman, Chem. Phys. Lett. 478, 150 (2009).

${ }^{8}$ A. Cuetos and B. Martínez-Haya, J. Chem. Phys. 129, 214706 (2008).

${ }^{9}$ S. Fraden, G. Maret, D. L. D. Caspar, and R. B. Meyer, Phys. Rev. Lett. 63, 2068 (1989).

${ }^{10}$ M. P. B. Van Bruggen, F. M. Van der Kooij, and H. N. W. Lekkerkerker, J. Phys. Condens. Matter 8, 9451 (1996).

${ }^{11}$ F. M. Van der Kooij and H. N. W. Lekkerkerker, J. Phys. Chem. B 102, 7829 (1998).

${ }^{12}$ F. M. Van der Kooij, K. Kassapidou, and H. N. W. Lekkerkerker, Nature (London) 406, 868 (2000).

${ }^{13}$ M. Wojcik and K. E. Gubbins, Mol. Phys. 53, 397 (1984).

${ }^{14}$ B. Martínez-Haya and A. Cuetos, Mol. Simul. 35, 1077 (2009).

${ }^{15}$ B. Martínez-Haya and A. Cuetos, J. Chem. Phys. 131, 074901 (2009). 
${ }^{16}$ B. Martínez-Haya and A. Cuetos, Phys. Rev. E 81, 020701 (2010).

${ }^{17}$ L. Filion, M. Marechal, B. van Oorschot, D. Pelt, F. Smallenburg, and M. Dijkstra, Phys. Rev. Lett. 103, 188302 (2009).

${ }^{18}$ T. Boublik and I. Nezbeda, Collect. Czech. Chem. Commun. 51, 2301 (1986).

${ }^{19}$ W. R. Cooney, S. M. Thompson, and K. E. Gubbins, Mol. Phys. 66, 1269 (1989).

${ }^{20}$ J. Šedlbauer, S. Labík, and A. Malijevsk, Phys. Rev. E 49, 3179 (1991).

${ }^{21}$ M. J. Maeso, J. R. Solana, and J. Amoros, Mater. Chem. Phys. 33, 134 (1993).

${ }^{22}$ B. M. Mulder, Mol. Phys. 103, 1411 (2005).

${ }^{23}$ F. Gamez, S. Lago, B. Garzon, P. J. Merkling, and C. Vega, Mol. Phys. 106, 1331 (2008).

${ }^{24}$ M. Marechal and M. Dijkstra, Phys. Rev. E 77, 061405 (2008).

${ }^{25}$ M. Marechal and M. Dijkstra, Phys. Rev. E 82, 031405 (2010).

${ }^{26}$ M. Parrinello and A. Rahman, Phys. Rev. Lett. 45, 1196 (1980).

${ }^{27}$ R. Najafabadi and S. Yip, Scripta Metall. 17, 1199 (1983).

${ }^{28}$ D. Frenkel and A. J. C. Ladd, J. Chem. Phys. 81, 3188 (1984).

${ }^{29}$ B. Widom, J. Chem. Phys. 39, 2808 (1963).

${ }^{30}$ M. A. Bates and D. Frenkel, Phys. Rev. E 57, 4824 (1998).

${ }^{31}$ P. Bolhuis and D. Frenkel, J. Chem. Phys. 106, 666 (1997).

${ }^{32}$ L. H. de la Peña, R. van Zon, J. Schofield, and S. B. Opps, J. Chem. Phys. 126, 074105 (2007).

${ }^{33}$ A. M. Ferrenberg and R. H. Swendsen, Phys. Rev. Lett. 61, 2635 (1988).

${ }^{34}$ R. Ni, S. Belli, R. van Roij, and M. Dijkstra, Phys. Rev. Lett. 105, 088302 (2010).

${ }^{35}$ A. Panagiotopoulos, Mol. Phys. 61, 813 (1987).

${ }^{36}$ E. Bianchi, P. Tartaglia, E. Zaccarelli, and F. Sciortino, J. Chem. Phys. 128, 144504 (2008).

${ }^{37}$ M. A. Bates and D. Frenkel, J. Chem. Phys. 110, 6553 (1999).

${ }^{38}$ A. Gavezzotti and G. R. Desiraju, Acta Crystallogr. Sec. B 44, 427 (1988).
${ }^{39}$ D. van der Beek, H. Reich, P. van der Schoot, M. Dijkstra, T. Schilling, R. Vink, M. Schmidt, R. van Roij, and H. Lekkerkerker, Phys. Rev. Lett. 97, 087801 (2006)

${ }^{40}$ H. Hemmen, N. I. Ringdal, E. N. De Azevedo, M. Engelsberg, E. L. Hansen, Y. Meheust, J. O. Fossum, and K. D. Knudsen, Langmuir 25, 12507 (2009).

${ }^{41}$ A. A. Verhoeff, R. H. J. Otten, P. van der Schoot, and H. N. W. Lekkerkerker, J. Phys. Chem. B 113, 3704 (2009).

${ }^{42}$ M. P. Allen, G. T. Evans, D. Frenkel, and B. M. Mulder, Adv. Chem. Phys. 86, 1 (1993).

${ }^{43}$ P. Virnau and M. Muller, J. Chem. Phys. 120, 10925 (2004).

${ }^{44}$ R. Eppenga and D. Frenkel, Mol. Phys. 52, 1303 (1984).

${ }^{45}$ A. Esztermann, H. Reich, and M. Schmidt, Phys. Rev. E 73, 011409 (2006).

${ }^{46} \mathrm{P}$. de Gennes and J. Prost, The Physics of Liquid Crystals (Oxford University Press, New York, 1995).

${ }^{47}$ S. Laschat, A. Baro, N. Steinke, F. Giesselmann, C. Hgele, G. Scalia, R. Judele, E. Kapatsina, S. Sauer, A. Schreivogel, and M. Tosoni, Angew. Chem., Int. Ed. 46, 4832 (2007).

${ }^{48}$ S. Sergeyev, W. Pisula, and Y. H. Geerts, Chem. Soc. Rev. 36, 1902 (2007).

${ }^{49}$ N. Boden, R. J. Bushby, and C. Hardy, Liq. Cryst. 1, 109 (1986).

${ }^{50}$ H. Bengs, O. Karthaus, C. Baehr, and J. H. Wendorff, Liq. Cryst. 10, 161 (1991).

${ }^{51}$ K. Praefcke, D. Singer, M. Ebert, and J. H. Wendorff, Liq. Cryst. 10, 147 (1991).

${ }^{52}$ K. Praefcke and J. D. Holbrey, J. Inclusion Phenom. Macrocyclic Chem. 24, 19 (1996).

${ }^{53}$ A. R. A. Palmans, J. A. J. M. Vekemans, R. A. Hikmet, H. Fischer, and E. W. Meijer, Adv. Mater. 10, 873 (1998).

${ }^{54}$ W. H. Press, S. A. Teukolsky, W. T. Vetterling, and B. P. Flannery, Numerical Recipes in C, 2nd ed. (Cambridge University Press, Cambridge, 1992). 